Article

\title{
Dynamic Evaluation of Circulating miRNA Profile in EGFR-Mutated NSCLC Patients Treated with EGFR-TKIs
}

\author{
Alessandro Leonetti 1,2,3 (D), Mjriam Capula ${ }^{3,4,5}$, Roberta Minari ${ }^{2}$ (D), Giulia Mazzaschi ${ }^{2}$, Alessandro Gregori ${ }^{3}$, \\ Btissame El Hassouni ${ }^{3}$, Filippo Papini ${ }^{3,4}$, Paola Bordi ${ }^{2}$, Michela Verzè ${ }^{2}$, Amir Avan ${ }^{3,6}$, Marcello Tiseo ${ }^{1,2, *}$ (D) \\ and Elisa Giovannetti $3,4, * \mathbb{D}$
}

1 Department of Medicine and Surgery, University of Parma, 43126 Parma, Italy; aleonetti@ao.pr.it

2 Medical Oncology Unit, University Hospital of Parma, 43126 Parma, Italy; rominari@ao.pr.it (R.M.); giulia.mazzaschi@studenti.unipr.it (G.M.); pbordi@ao.pr.it (P.B.); mverze@ao.pr.it (M.V.)

3 Department of Medical Oncology, Amsterdam UMC, VU University, Cancer Center Amsterdam, 1081 HV Amsterdam, The Netherlands; m.capula@fpscience.it (M.C.); a.gregori@amsterdamumc.nl (A.G.); b.elhassouni@amsterdamumc.nl (B.E.H.); f.papini@student.vu.nl (F.P.); AvanA@mums.ac.ir (A.A.)

4 Fondazione Pisana per la Scienza, San Giuliano, 56017 Pisa, Italy

5 Institute of Life Sciences, Sant'Anna School of Advanced Studies, 56100 Pisa, Italy

6 Metabolic Syndrome Research Center, Mashhad University of Medical Sciences, 13131-99137 Mashhad, Iran

* Correspondence: marcello.tiseo@unipr.it (M.T.); elisa.giovannetti@gmail.com (E.G.)

check for updates

Citation: Leonetti, A.; Capula, M.; Minari, R.; Mazzaschi, G.; Gregori, A.; El Hassouni, B.; Papini, F.; Bordi, P.; Verzè, M.; Avan, A.; et al. Dynamic Evaluation of Circulating miRNA Profile in EGFR-Mutated NSCLC Patients Treated with EGFR-TKIs. Cells 2021, 10, 1520. https://doi.org/ $10.3390 /$ cells10061520

Academic Editor: Steven G. Gray

Received: 18 May 2021

Accepted: 11 June 2021

Published: 16 June 2021

Publisher's Note: MDPI stays neutral with regard to jurisdictional claims in published maps and institutional affiliations.

Copyright: (c) 2021 by the authors. Licensee MDPI, Basel, Switzerland. This article is an open access article distributed under the terms and conditions of the Creative Commons Attribution (CC BY) license (https:// creativecommons.org/licenses/by/ $4.0 /)$.
Abstract: Background: Resistance to EGFR-TKIs constitutes a major challenge for the management of EGFR-mutated NSCLC, and recent evidence suggests that deregulation of specific microRNAs (miRNAs) may influence resistance to targeted agents. In this retrospective study, we explored the role of specific plasmatic miRNAs (miR-21, miR-27a and miR-181a) as a surrogate for predicting EGFR-TKI performance in EGFR-mutated NSCLC patients. Methods: Plasma samples of 39 advanced EGFR-mutated NSCLC patients treated with EGFR-TKIs were collected at different points in time and miRNA levels were assessed by RT-PCR. Results: Higher basal values of miR-21 were reported in patients who achieved a partial/complete response (PR/CR) compared to those with stability/progression of disease (SD/PD) $(p=0.011)$. Along the same line, patients who experienced a clinical benefit lasting at least six months displayed higher basal levels of circulating miR-21 $(p=0.039)$. However, dynamic evaluation of miRNA values after two months from the start of EGFR-TKI treatment showed that patients who experienced SD had an increase in miR-21 levels (Fold Change $[F C]=2.6)$ compared to patients achieving PR/CR $(p=0.029)$. The same tendency was observed for miR-27a $(F C=3.1)$ and miR-181a $(F C=2.0)$, although without reaching statistical significance. Remarkably, preclinical studies showed an increase in miR-21 levels in NSCLC cells that became resistant after exposure to EGFR-TKIs. Conclusions: Our study provides interesting insights on the role of circulating miRNAs, in particular miR-21, and their dynamic change over time in predicting EGFR-TKI response in EGFR-mutated NSCLC.

Keywords: miRNA; EGFR-mutated NSCLC; response to EGFR-TKI; targeted therapy

\section{Introduction}

Lung cancer is the second most common malignancy and represents the leading cause of cancer-related death worldwide [1]. Targeted therapy revolutionized the treatment paradigm of advanced non-small-cell lung cancer (NSCLC) in the presence of druggable driver mutations, achieving outstanding results in selected populations. Among driver targetable mutations, Epidermal Growth Factor Receptor (EGFR) mutations can be found in approximately $10-16 \%$ of NSCLC patients from Western countries, and this percentage is even larger when only considering Asian patients (up to 50\%) [2].

In the presence of a sensitizing EGFR mutation, advanced NSCLC patients are treated frontline with selective tyrosine kinase inhibitors (TKI), among which gefitinib and erlotinib are first-generation TKIs, whereas afatinib and dacomitinib are second-generation 
irreversible inhibitors. However, despite initial high responses to these drugs, the tumor often implements mechanisms to escape the pathway blockade, ultimately resulting in disease progression [3]. Moreover, given the fact that primary resistance to EGFR-TKIs exists in a portion of patients in the presence of a sensitizing EGFR mutation, it is critical to detect potential biomarkers that can help identify the subgroup of patients with primary resistance to EGFR-TKIs therapy.

Over $50 \%$ of acquired resistance to first- and second-generation TKIs is caused by the onset of 'gatekeeper' mutation T790M, which compromises the binding of the abovementioned compounds to EGFR, and increases the receptor affinity for ATP [4]. Thirdgeneration TKI osimertinib can overcome this resistance mechanism, and it has proven to be effective in EGFR-mutated NSCLC irrespective of T790M status, but the clinical benefit would be limited by further emergence of resistance $[5,6]$. Hence, to date, earlier identification of resistance mechanisms as well as the development of new strategies to get through the limitations of the EGFR blockade alone are needed.

MicroRNAs (miRNAs) are 18-25 nucleotides in length, single-stranded non-coding RNAs that function as post-transcriptional regulators of gene expression. Overall, gene silencing mediated by miRNAs is reflected in the regulation of different cellular processes, including cell differentiation, proliferation, apoptosis, and stem cell self-renewal. With respect to carcinogenesis, miRNAs can act as either oncogenes or tumor suppressors, depending on the cellular context and the multiple target genes affected by miRNA silencing [7]. Moreover, accumulating evidence suggests that deregulation of specific miRNAs may also influence cancer cell resistance to conventional chemotherapy and novel targeted agents.

Regarding EGFR-mutated NSCLC, recent evidence suggests that key miRNAs can deregulate pivotal pathways involved in cell survival, metabolism, epithelial-to-mesenchymal transition (EMT), and apoptosis. More interestingly, studies denoted miRNAs as modulators of response to TKIs in EGFR-mutated NSCLC, both in vitro and in vivo, thus conditioning both primary and acquired resistance [8]. Furthermore, miRNA expression profiles could serve as biomarkers for predicting patients' prognosis and response to the targeted treatments. Indeed, growing evidence suggests that dynamic evaluation of miRNA levels, detected in patients' plasma samples, could represent an emerging useful tool for the monitoring of EGFR-TKI therapy in EGFR-driven NSCLC, but this approach has not yet been implemented in the clinical practice [9].

Among different miRNAs, different studies have pointed out miR-21, miR-27a, and miR-181a as potentially responsible for resistance to EGFR-TKIs in EGFR-mutated NSCLC. In particular, miR-21 and miR-27a were found to be significantly overexpressed in plasma samples of EGFR-mutated NSCLC patients with primary resistance to TKIs compared to the sensitive group [10]. In addition, miR-181a contributed to gefitinib resistance in lung cancer cells by targeting GAS7, and was upregulated in gefitinib-resistant cells compared to gefitinib-sensitive cells [11]. Given the preclinical and clinical evidence, a deeper understanding of the clinical implications of miR-21, miR-27a, and miR-181a is warranted. Furthermore, a dynamic evaluation of these miRNAs in blood samples of EGFR-mutated patients undergoing TKIs is lacking. Hence, we designed a research study to explore the role of candidate plasma miRNAs (miR-21, miR-27a, and miR-181a) as a surrogate for predicting EGFR-TKIs performance in advanced EGFR-mutated NSCLC patients (DynaMiR Study).

\section{Materials and Methods}

\subsection{Patients Population}

The present research was performed on aliquots of plasma samples that were collected during a previously approved research protocol (DiNAmic study; protocol Version 1, 28 February 2015) at the Department of Medical Oncology of the University Hospital of Parma. Patients must have received EGFR-TKI treatment (gefitinib, erlotinib, or afatinib) 
for advanced EGFR-mutated NSCLC. Only patients with sensitizing EGFR mutations were enrolled; patients with non-sensitizing EGFR mutations (i.e., ins20) were excluded.

Patients enrolled in the study underwent a blood sample collection at different points in time. A specific consent form for the collection of blood samples was presented to each patient at the time of enrolment into the DiNAmic study (before any specific procedure) and was signed by the patients who decided to take part in the study.

The following clinico-pathological information was collected at baseline as per clinical practice: age, ECOG PS, comorbidities of the patients, smoking history, number and type of metastatic sites, and presence of brain metastases. Plasma analyses were conducted at the Laboratory Medical Oncology at the Amsterdam University Medical Centers (Amsterdam UMC) VU University, Amsterdam, The Netherlands; and at the Fondazione Pisana per la Scienza, Pisa, Italy.

\subsection{Plasma Collection and RNA Extraction}

Patients underwent collection of $6 \mathrm{~mL}$ of blood in Ethylenediaminetetraacetic acid (EDTA) tubes. Samples were collected at the beginning of TKI treatment (t0); after two months (t1); at the time of first radiological evaluation (t2); and at the time of radiologically documented progression of disease, according to RECIST 1.1 criteria (tPD). Tubes were centrifuged twice for $10 \mathrm{~min}$ at $2000 \mathrm{rcf}$ within $2 \mathrm{~h}$ after collection, and separated plasma was frozen at $-80^{\circ} \mathrm{C}$ until RNA extraction.

RNA was extracted from $200 \mu \mathrm{L}$ plasma using miRNeasy Serum/Plasma Kit (Qiagen, Hilden, Germany) according to the manufacturer's instructions, with a minor modification: $1 \mu \mathrm{L}$ of miRNeasy Serum/Plasma Spike-in control (20 fmol cel-miR-39-3p synthetic RNA Spike-In) was added before the addition of Buffer RPL and 1.25 $\mu \mathrm{L}$ of MS2 bacteriophage carrier RNA (Roche, Castle Hill, NSW, Australia).

\section{3. miRNA Quantification}

RNA samples were reverse-transcribed to cDNA using a cDNA synthesis kit (Qiagen, Hilden, Germany) according to the manufacturer's instructions. The miRCURY LNA SYBR Green PCR Kit and RT primers (hsa-miR-21-5p, Cat. ID: YP00204230; hsa-miR-27a-3p, Cat. ID: YP00206038; hsa-miR-181a-5p, Cat. ID: YP00206081; and cel-miR-39-3p, Cat. ID: YP00203952), were used for RT-qPCR on CFX96 Real-time System (Biorad, Hercules, CA, USA), in line with the accompanying protocols.

Cel-miR-39-3p was used as a spike-in control to normalize the variation in RNA extraction, and also as a reference for the relative quantification instead of internal controls. Cyclee thresholds (Cts) were automatically calculated with CFS Manager Software (Biorad, Hercules, CA, USA).

Technical duplicates were performed for all miRNAs. MiRNA expression was normalized by subtracting the average $\mathrm{Ct}$ value of the cel-miR39 from the average $\mathrm{Ct}$ value of the miRNA of interest, to obtain the $\Delta \mathrm{Ct}$. The miRNA value was expressed as $2^{-\Delta \mathrm{Ct}}$. Fold-change (FC) was expressed as $2^{-\Delta \Delta C t}$, according to the $\Delta \Delta \mathrm{Ct}$ method [12]. As consistent with previous reports, FC $\geq 2$ denoted an increase in miRNA expression, FC $\leq 0.5$ denoted a decrease in miRNA expression, and $0.5<\mathrm{FC}<2$ denoted a stability in miRNA expression [13,14].

\subsection{Cell Culture}

The human NSCLC cell lines A549, NCI-H1299, NCI-H23, NCI-H3255, NCI-H1650, and HCC-827 were purchased from ATCC (Manassas, VA, USA) and cultured as recommended. HCC-827GR5 and PC9 were a kind gift from Dr. Pasi A. Jänne, Harvard University, Boston, MA [15]. Cells were grown as a monolayer in $75 \mathrm{~cm}^{2}$ flasks (Costar, Cambridge, MA, USA) at $37^{\circ} \mathrm{C}$ in $5 \% \mathrm{CO}_{2}$ and $95 \%$ air. The NSCLC cell lines used in this study had been previously characterized for EGFR and KRAS mutations, and were tested for their authentication by PCR profiling using short tandem repeats (STR), at BaseClear (Leiden, The Netherlands). 


\subsection{Inhibition of Cell Proliferation}

Cell growth inhibition was assessed by a sulforhodamine B (SRB) assay according to the NCI protocol, as described previously [16]. Cells were plated at $10^{4}$ cells/well, and each drug (gefitinib or afatinib) was tested in triplicate. Growth inhibition was expressed as the percentage of control (0.1\% DMSO-treated cells) absorbance (corrected for absorbance before drug exposure). A volume of $25 \mu \mathrm{L}$ of ice-cold $50 \%(w / v)$ trichloroacetic acid was added after $72 \mathrm{~h}$ treatment. The $50 \%$ inhibitory concentration of cell growth (IC50) was calculated by non-linear least squares curve fitting (GraphPad PRISM, Intuitive Software for Science, San Diego, CA, USA).

\subsection{Establishment and Genetic Characterization of Gefitinib and Afatinib-Resistant Cells}

In vitro acquired resistance to gefitinib and afatinib was modeled by applying doseescalation (up to $1 \mu \mathrm{M}$ ) of these compounds to PC9 cells. Afatinib resistant cells were exposed to a continuous (PC9-AR1) or a pulse (PC9-AR2) exposure. Genomic DNA was extracted using the Ambion ${ }^{\circledR}$-RecoverAll kit (Life Technologies, Breda, The Netherlands). The quantity and purity of the extracted DNA were assessed at 260-280 nm with the NanoDrop ${ }^{\circledR}$-1000-Detector (NanoDrop-Technologies, Wilmington, NC, USA), and EGFR, KRAS, BRAF, and PIK3CA mutational statuses were determined as previously described [17]. Nomenclature of EGFR mutations is reported in the Appendix A.

\subsection{Quantitative PCR Analysis of miR-21 in NSCLC Cells}

RNA was extracted according to the Trizol-chloroform protocol and the miR-21 basal expression, and its possible modulation in cells which became resistant to gefitinib and afatinib was assessed by quantitative PCR using the $\Delta \Delta C t$ method [18].

\subsection{Effects of miR-21 Transfection in Resistant NSCLC Cells}

The effect of miR-21 on the inhibition of cell growth was evaluated by transfecting the NSCLC resistant cells with miR-21 antisense oligonucleotides (anti-miR-21), purchased from Ambion-Applied Biosystems (Assay ID, AM10206), at $30 \mathrm{nM}$ final concentration. Cells were also incubated with miRNA negative controls and FAM-labeled anti-miR (AmbionApplied Biosystems, Waltham, MA, USA), as described previously [18].

\subsection{EGFR and Akt Phosphorylation Assays}

To study whether the expression of miR-21 correlated with EGFR activity, as well as whether resistance to EGFR-TKIs was associated to modulation of EGFR downstream pathways, cells were harvested when they were in exponential growth. After protein extraction from cell pellets, EGFR phosphorylation at the tyrosine residue at position 1173 (EGFR [pY1173]) and Akt phosphorylation at serine residue 473 (Akt [pS473]) were evaluated with specific ELISA assays (BioSource International, Camarillo, CA, USA), and were normalized respectively to the total EGFR, Akt and protein content, as described previously [19].

\subsection{Statistical Analysis}

Statistical analysis was performed using SPSS Statistical Software version 27.0 (SPSS, IBM, USA) and GraphPad Prism Software version 6 (Intuitive Software for Science, San Diego, CA, USA).

Cq values were expressed as mean values $\pm \mathrm{SD}$. For each miRNA of interest at the baseline, patients were divided into two groups with respect to the median expression value of the miRNA (high vs. low). Wilcoxon and Mann-Whitney tests were used to compare the two groups of paired and unpaired data, respectively. The Kruskal-Wallis test was employed for the analysis of group differences. Progression-free survival (PFS) and overall survival (OS) were calculated from the start of EGFR-TKI treatment until radiological confirmed progression of disease or death, respectively, using the Kaplan- 
Meier method. Statistical differences were assessed using the log-rank test. All $p$-values were two sided, and $p<0.05$ was used to indicate a statistically significant difference.

All in vitro experiments were performed in triplicate and repeated at least twice. Data were expressed as mean values \pm SEM and analyzed using the two-tailed Student's t-test or ANOVA, followed by the Bonferroni's multiple comparison test using GraphPad Prism Software version 6 (Intuitive Software for Science, San Diego, CA, USA). The level of significance was $p<0.05$.

\section{Results}

\subsection{Patient Characteristics}

The study population involved 39 patients. Baseline patient characteristics are summarized in Table 1.

Table 1. Patient Characteristics.

\begin{tabular}{|c|c|}
\hline Patient Characteristics & Number (\%) \\
\hline \multicolumn{2}{|l|}{ Gender } \\
\hline Male & $16(41)$ \\
\hline Female & $23(59)$ \\
\hline \multicolumn{2}{|l|}{ Age at Diagnosis } \\
\hline$<65$ years & $18(46)$ \\
\hline$\geq 65$ years & $21(54)$ \\
\hline \multicolumn{2}{|l|}{ Smoking Status } \\
\hline Current/former & $18(46)$ \\
\hline Never & $21(54)$ \\
\hline \multicolumn{2}{|l|}{ ECOG PS } \\
\hline 0 & $21(54)$ \\
\hline 1 & $16(41)$ \\
\hline 2 & $2(5)$ \\
\hline \multicolumn{2}{|l|}{ Brain Mets at Baseline } \\
\hline No & $24(61)$ \\
\hline Yes & $15(39)$ \\
\hline \multicolumn{2}{|l|}{ Liver Mets at Baseline } \\
\hline No & $34(87)$ \\
\hline Yes & $5(13)$ \\
\hline \multicolumn{2}{|l|}{ Bone Mets at Baseline } \\
\hline No & $24(61)$ \\
\hline Yes & $15(39)$ \\
\hline \multicolumn{2}{|l|}{ Number of Metastatic Sites } \\
\hline$<3$ & $22(56)$ \\
\hline$\geq 3$ & $17(44)$ \\
\hline \multicolumn{2}{|l|}{ EGFR Mutation } \\
\hline Ex19del & $19(49)$ \\
\hline L858R & $16(41)$ \\
\hline G719X + S768I & $1(2.5)$ \\
\hline Ex19del + T790M & $1(2.5)$ \\
\hline $\mathrm{L} 858 \mathrm{R}+\mathrm{S} 768 \mathrm{I}$ & $1(2.5)$ \\
\hline S768I & $1(2.5)$ \\
\hline \multicolumn{2}{|l|}{ EGFR-TKI } \\
\hline Gefitinib & $23(59)$ \\
\hline Erlotinib & $7(18)$ \\
\hline Afatinib & $9(23)$ \\
\hline \multicolumn{2}{|l|}{ Line of Treatment } \\
\hline First-line & $37(95)$ \\
\hline Second-line & $2(5)$ \\
\hline \multicolumn{2}{|l|}{ Best Response to EGFR-TKI } \\
\hline CR & $1(2.5)$ \\
\hline PR & $28(72)$ \\
\hline SD & $6(15.5)$ \\
\hline PD & $4(10)$ \\
\hline
\end{tabular}


Concerning the correlation between clinico-pathological characteristics and survival outcome, Kaplan-Meier curves showed that patients with brain metastases at baseline had a shorter OS than patients without brain metastases (median OS: 14.0 vs. 37.3 months [Hazard Ratio (HR), 2.49; 95\% CI: 1.13-5.48; $p=0.023$ ]) (Figure S1A). Along the same line, patients who had liver metastases at baseline displayed shorter OS than patients without liver metastatic involvement (median OS: 11.6 vs. 34.1 months [HR, 3.48; 95\% CI: 1.29-9.41; $p=0.023$ ], $p=0.014$ ) (Figure S1B). None of the other basal features (sex, age, smoking status, number of metastatic sites, and type of EGFR mutation) were significantly associated with either PFS or OS. No correlations were observed between baseline patient characteristics and either response to EGFR-TKIs (PR/SD vs. PD) or duration of clinical benefit ( $\geq 6$ months vs. $<6$ months) (data not shown).

\subsection{Basal miRNA Expression and Correlation with Clinical Features}

Clinical characteristics of patients (gender, smoking status, bone metastasis, central nervous system metastasis, pleural effusion, liver metastasis, number of metastatic sites, and type of EGFR mutation) were subsequently correlated with basal values of miR-21, miR-27a and miR-181a. Among all features, patients aged $<65$ years had significantly higher basal values of miR-21 compared to the counterpart $(p=0.044)$. No further differences in basal miRNA expression were observed according to other clinical features (Figures S2 and S3). When looking at relevant comorbidities, we did not find any difference in basal miR-21, miR-27a and miR-181a according to the presence/absence of comorbidity (Table S2).

\subsection{Basal miRNA Expression and Correlation with Clinical Outcome}

All the enrolled patients had baseline samples collected before the start of EGFR-TKI treatment. As reported in Figure S4, basal levels of the three candidate miRNAs were significantly correlated to each other (miR-21 vs. miR-27a: $\mathrm{R}=0.922, p<0.001$; miR-21 vs. miR-181a: $\mathrm{R}=0.789, p<0.001$; miR-27a vs. miR-181a: $\mathrm{R}=0.732, p<0.001)$. This is in agreement with previous studies showing that miR-21 and miR-27a acted as cooperative repressors of a network of tumor suppressor genes that included PDCD4, BTG2, and NEDD4L [20].

In order to find a putative correlation between basal miRNA values and clinical outcomes, patients were divided into two groups with respect to the median expression value of the investigated miRNA (High vs. Low). Basal values of miR-21, miR-27a and miR-181a were not significantly correlated with PFS (miR-21 Low vs. High: median PFS 11.1 vs. 9.0 months, $p=0.780$; miR-27a Low vs. High: median PFS 11.1 vs. 9.0 months, $p=0.334$; miR-181a Low vs. High: median PFS 13.1 vs. 8.5 months, $p=0.152$ ) (Figure 1A). The same trend was observed for the OS (miR-21 Low vs. High: median OS 24.2 vs. 37.3 months, $p=0.383$; miR-27a Low vs. High: median OS 24.2 vs. 37.3 months, $p=0.224$; miR-181a Low vs. High: median OS 34.1 vs. 16.8 months, $p=0.201$ ) (Figure 1B).

Basal differential expression of the candidate miRNAs was evaluated on the basis of the best objective response to EGFR-TKI treatment. When patients were stratified according to their best response (partial response/complete response $[\mathrm{PR} / \mathrm{CR}]$ vs. stable disease/progression of disease [SD/PD]), patients who achieved PR/CR as their best response had a significantly higher basal value of miR-21 than patients who achieved $\mathrm{SD} / \mathrm{PD}(p=0.011)$ (Figure 2A). Dividing the patients according to the duration of clinical benefit ( $\geq 6$ months vs. $<6$ months), patients who achieved a clinical benefit $\geq 6$ months had a higher basal value of miR-21 than patients with clinical benefit $<6$ months $(p=0.039)$ (Figure 2B). 
A
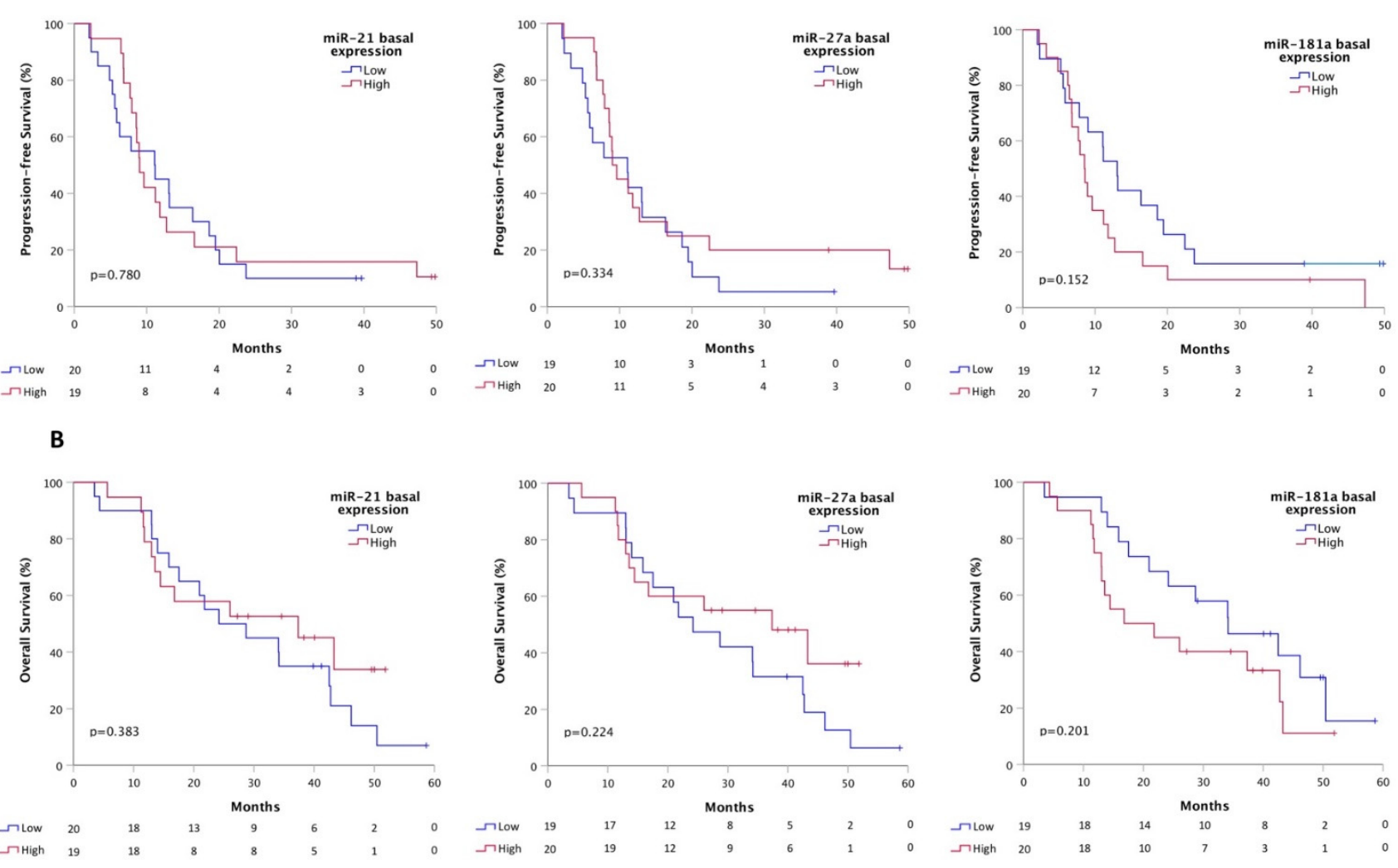

Figure 1. Kaplan-Meier curves for basal miRNA expression and correlation with PFS (A) and OS (B).
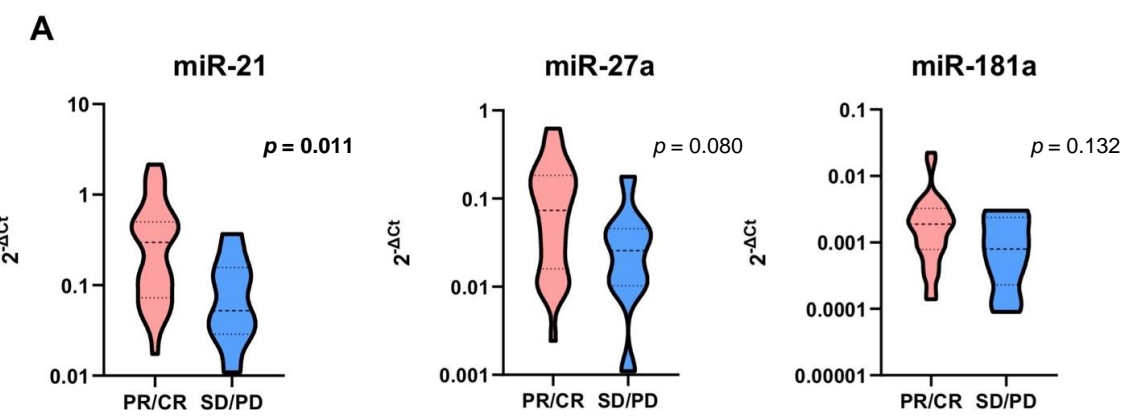

\section{B}

miR-21

miR-27a

miR-181a
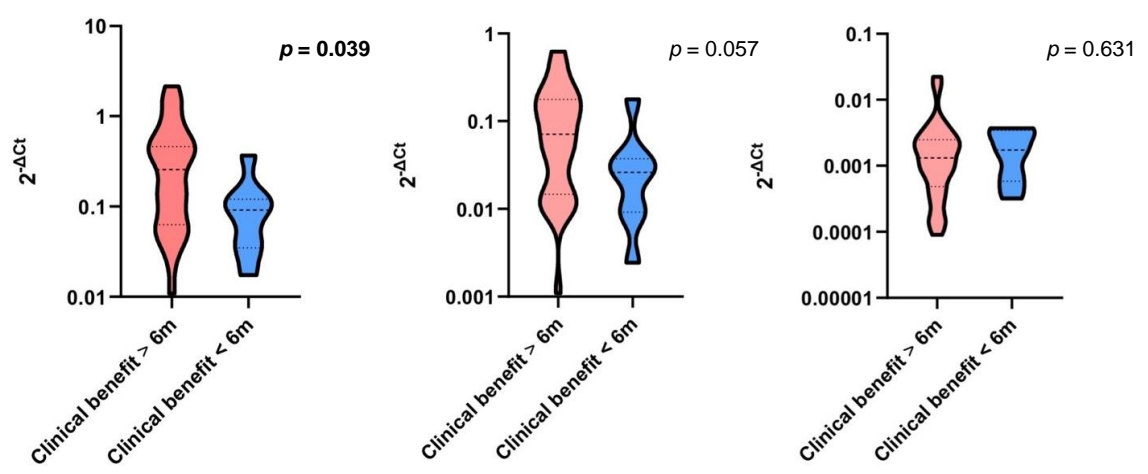

Figure 2. Basal miRNA expression and correlation with EGFR-TKI response (A) and duration of clinical benefit (B). Significant $p$ values are in bold. Dashed lines represent the median values. Dotted lines represent the upper and lower quartiles. 
When patients were stratified according to basal miRNA expression, those who had basal overexpression of either miR-21 or miR-27a were more likely to achieve a durable clinical benefit $\geq 6$ months (Fisher's exact test $p=0.044$ and $p=0.020$, respectively) (Table 2).

Table 2. Correlation between basal miRNA values and clinical benefit to EGFR-TKI.

\begin{tabular}{cccc}
\hline $\begin{array}{c}\text { Basal miRNA } \\
\text { Expression }\end{array}$ & $\begin{array}{c}\text { Clinical Benefit } \\
<6 \text { Months }\end{array}$ & $\begin{array}{c}\text { Clinical Benefit } \\
\geq 6 \text { Months }\end{array}$ & $p$ Values \\
\hline miR-21 low (\%) & $7(35 \%)$ & $13(65 \%)$ & 0.044 \\
miR-21 high (\%) & $1(5 \%)$ & $18(95 \%)$ & 0.020 \\
\hline miR-27a low (\%) & $7(37 \%)$ & $12(63 \%)$ & 0.451 \\
miR-27a high (\%) & $1(5 \%)$ & $19(95 \%)$ & $14(74 \%)$ \\
\hline miR-181a low (\%) & $5(26 \%)$ & $17(85 \%)$ & 0 \\
\hline miR-181a high $(\%)$ & $3(15 \%)$ & & 0 \\
\hline
\end{tabular}

\subsection{Modulation of miRNA Expression in Patients Treated with EGFR-TKIs}

Modulation of basal miRNA expression was evaluated on blood samples collected after two months from the beginning of TKI treatment and prior to the first radiological assessment, labeled as ' $t 1$ ', in order to correlate early variations of circulating miRNAs to differential EGFR-TKI responses.

From the enrolled patients, 24 patients $(62 \%)$ had ' $t 1$ ' samples available for this analysis and, among them, no one experienced PD as best response. Patients were then stratified according to their best response (PR/CR vs. SD) and the duration of clinical benefit ( $\geq 6$ months vs. $<6$ months).

Statistically significant differences in FC were observed for miR-21 at $\mathrm{t} 1$ compared to baseline according to the type of best response to EGFR-TKI (PR/CR vs. SD): miR-21 median FC at $\mathrm{t} 1$ in PR/CR vs. SD: 0.8 vs. 2.6, $p=0.029$. No statistically significant difference in FC was observed for miR27a and miR-181a at $\mathrm{t} 1$ compared to baseline according to the type of best response to EGFR-TKI, even though a trend towards an increase in miR-27a and miR-181a at $\mathrm{t} 1$ was observed for patients with SD compared to PR/CR: miR-27a median FC at $\mathrm{t} 1$ in PR/CR vs. SD: 0.8 vs. 3.1, $p=0.061$; miR-181a median FC at $\mathrm{t} 1$ in PR/CR vs. SD: 1.2 vs. $2.0, p=0.156$ (Figure $3 \mathrm{~A}$ ).

A
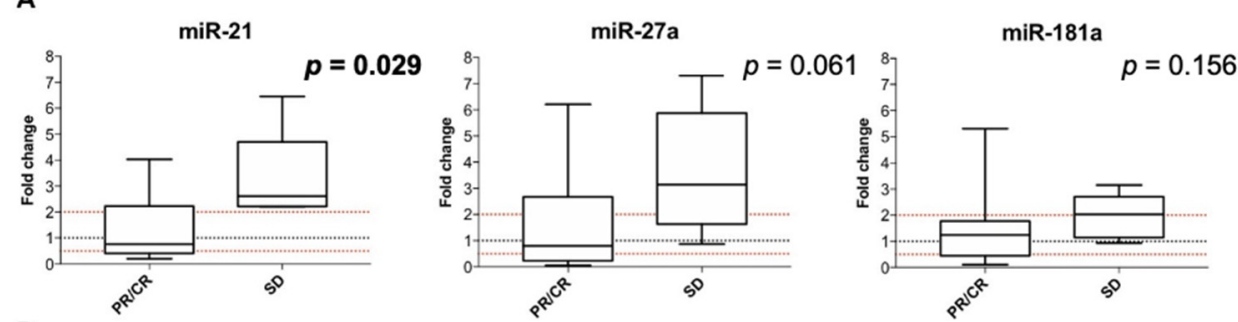

$\begin{array}{lll}B & \text { miR-21 } \quad p=0.082\end{array}$
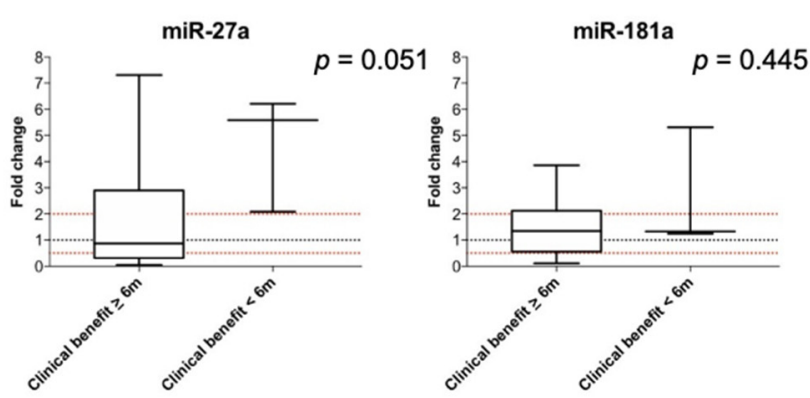

Figure 3. Modulation of miRNA expression at $\mathrm{t} 1$ and correlation with EGFR-TKI response (A) and duration of clinical benefit (B). Significant $p$ values are in bold. The box plot represents the upper to lower quartiles, the whiskers are the minimum and maximum values, and the black lines represent the median values. 
No statistically significant difference in FC was observed for miRNAs compared to baseline according to the clinical benefit to EGFR-TKI, even though a trend towards an increase in miR-21 and miR-27a at $\mathrm{t} 1$ was observed for patients with clinical benefit $<6$ months compared to patients with clinical benefit $\geq 6$ months: miR-21 median FC at 11 in clinical benefit $\geq 6$ months vs. $<6$ months: 0.8 vs. $3.4, p=0.082$; miR-27a median FC at t1 in clinical benefit $\geq 6$ months vs. $<6$ months: 0.9 vs. $5.6, p=0.051$; miR-181a median FC at $\mathrm{t} 1$ in clinical benefit $\geq 6$ months vs. $<6$ months: 1.3 vs. $1.3, p=0.445$ (Figure $3 \mathrm{~B}$ ).

Since an increase in miRNAs at $\mathrm{t} 1$ denoted patients who were likely to achieve $\mathrm{SD}$ as their best response rather than PR, as well as a limited clinical benefit, patients were further stratified according to the increase $(\mathrm{FC} \geq 2)$ vs. decrease/stability $(\mathrm{FC}<2)$ of miRNAs at $\mathrm{t} 1$, and groups were compared in terms of PFS and OS. No statistically significant differences were observed between patients who had an increase in miRNAs compared to those who had a decrease/stability at $\mathrm{t} 1$, both in terms of PFS and OS (data not shown).

\subsection{Modulation of miRNA Expression at the Time of First Radiological Evaluation}

Change in basal miRNA expression was evaluated on blood samples collected at the time of first radiological assessment, labeled as ' $t 2$ '. Thirty-two patients $(82 \%)$ had ' $t 2$ ' samples available for this analysis. Patients were stratified according to their best response (PR/CR vs. SD/PD) and the duration of clinical benefit ( $\geq 6$ months vs. $<6$ months).

No statistically significant difference in FC was observed for miRNAs at $\mathrm{t} 2$ compared to baseline according to both the type of best response and clinical benefit to EGFR-TKI (Figure S5). No statistically significant differences were observed between patients who had an increase in miRNAs compared to those who had a decrease/stability at t2, both in terms of PFS and OS (data not shown).

\subsection{Modulation of miRNA Expression at the Time of Progression of Disease}

Change from basal miRNA expression was evaluated from blood samples collected at the time of progression to EGFR-TKI treatment, labeled as 'tPD'. Among the 35 patients who experienced disease progression, one patient did not have a tPD sample available and was excluded from this analysis. miR-181a levels at the time of progression were stable compared to the basal values, and this data was statistically significant (median FC: $1.7, p=0.012$ ). miR-21 and miR-27a levels were stable compared to the relative basal values, although without reaching statistical significance (median FC miR-21: 1.1, $p=0.270$; median FC miR-27a: 1.3, $p=0.397$, respectively) (Figure 4).

$\operatorname{miR}-21$

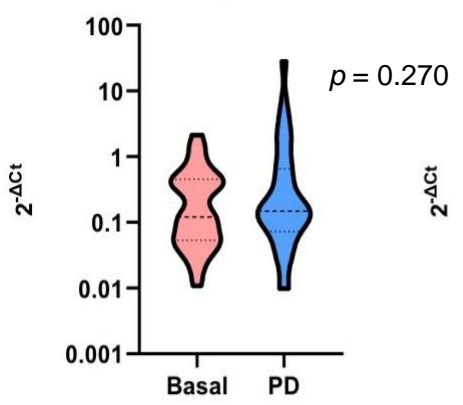

$\operatorname{miR}-27 a$

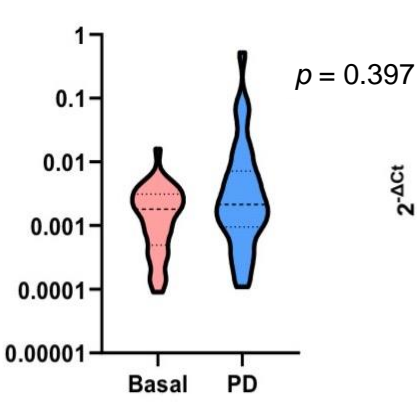

$\operatorname{miR}-181 a$

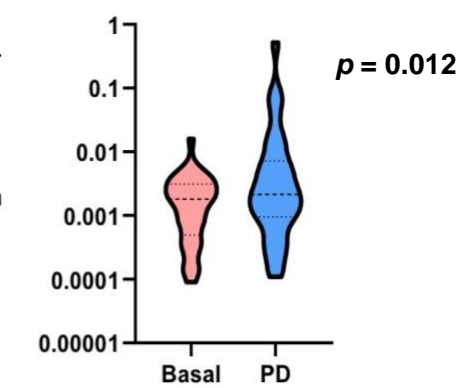

Figure 4. Modulation of miRNA expression at the time of progression to EGFR-TKI compared to baseline. Significant $p$ values are in bold. Dashed lines represent the median values. Dotted lines represent the upper and lower quartiles.

3.7. Correlation between miR-21 Expression, Chemosensitivity and Phosphorylated-EGFR Levels in NSCLC Cell Lines

The expression of miR-21 showed a large heterogeneity, and we evaluated whether the different sensitivity to drug treatment may be related to variable cellular miR-21 expression 
profiles (Table S1) and to phospho-EGFR in a panel of NSCLC cells characterized by their EGFR status.

The cells harboring EGFR activating mutations (NCI-H3255, HCC-827 and PC9) were the most sensitive to all the EGFR-TKIs, and had the highest expression levels of both miR-21 and phospho-EGFR (Figure 5A).

A

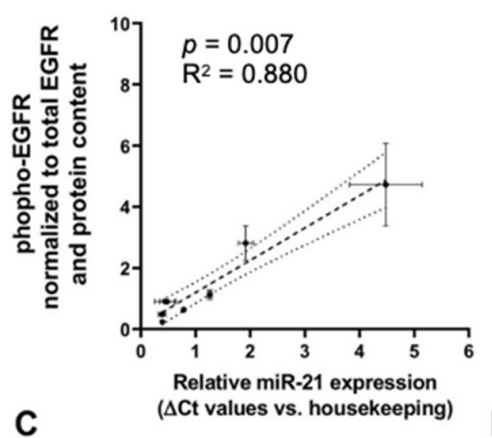

C

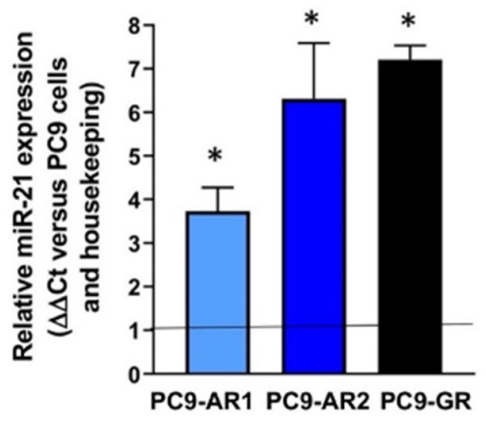

B
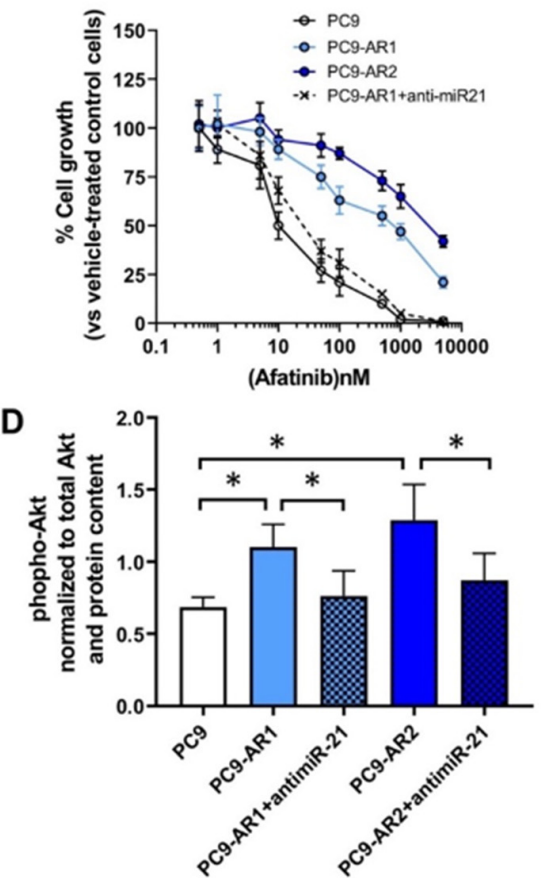

Figure 5. In vitro studies on the impact of miR-21 on phospho-EGFR and resistance to EGFR-TKIs: (A) Spearman correlation between miR-21 expression and phospho-EGFR levels in A549, NCI-H1299, NCI-H1703, NCI-H23, NCI-H1650, HCC827GR5, NCI-H3255, HCC827 and PC9 cells; (B) Inhibition of cell growth by afatinib in PC9 cells and the resistant cells PC9-AR1, PC9- AR2 and PC9-AR1 cells transfected with anti-miR-21; (C) Modulation of miR-21 expression in PC9 cells resistant to afatinib and gefitinib ( $p<0.05$ compared to wild type cells); (D) Modulation of phospho-Akt expression in PC9 cells resistant to afatinib and gefitinib cells transfected with anti-miR-21 or scramble negative controls $\left({ }^{*} p<0.05\right)$.

Conversely, the cells harboring both EGFR mutations and PTEN exon-9 loss or cMET amplification (i.e., NCI-H1650 and HCC827 cells) had $\mathrm{IC}_{50}$ and miR-21 values similar to the cells with wild-type EGFR status. No correlation was found between KRAS mutations and either miR-21 expression or drug sensitivity.

Next, we evaluated the modulation of miR-21 expression in the clones selected for their resistance to gefitinib and afatinib. After approximately six months, resistant clones emerged which were over 100-fold less sensitive to the drug (Figure 5B) than the parental cell line. Melting PCR and sequencing analyses were performed to select clones which did not have additional mutations in EGFR (exons 18-21), KRAS (exons 2-3), BRAF (exon 15) and PIK3CA (exons 9 and 20) genes. Interestingly, in all these clones, we observed a significant increase of miR-21 (of 2.7-3.8-fold, $p<0.05$ ), as reported in Figure 5C.

In order to further explore the role of miR-21 in the sensitivity of resistant cells, the PC9AR1 cells were transfected with miR-21-specific antisense inhibitors. Transfection efficiency was evaluated by analysis of fluorescent microscope images of cells transfected $24 \mathrm{~h}$ before with specific FAM-dye anti-miR oligonucleotides, showing at least $70 \%$ transfection efficiency, with $>90 \%$ cell viability. Furthermore, quantitative PCR analysis of miR-21 after anti-miR21 transfection showed a 2-fold reduction of miR-21 expression compared 
to cells transduced with negative controls (data not shown). The reduced expression of miR-21 was associated with a partial rescue of the sensitivity to afatinib, with more than 50-fold reduction of the IC50 in the PC9-AR1, suggesting that the aberrant expression of this miRNA correlated to drug resistance (Figure 5B). Similar results were observed for PC9-AR2 cells (data not shown).

To investigate the effects of miR-21 on EGFR downstream pathways, we evaluated the phosphorylation status of Akt in the wild-type/control, resistant and anti-miR21 transfected cells. The resistant cells were characterized by a significant increase of the phopho-Akt/Akt ratio ranging from +61 to $+88 \%$ in the PC9-AR1 and PC9-AR2 cells, respectively. In contrast, transfection with anti-miR-21 significantly reduced the activation status of Akt, with a pAkt/Akt ratio from 1.101 to $0.763 \mathrm{U} / \mathrm{ng}$ in PC9-AR1 and from 1.289 to $0.872 \mathrm{U} / \mathrm{ng}$ in PC9-AR2 cells (Figure 5D).

\section{Discussion}

In the present study, three candidate circulating miRNAs (miR-21, miR-27a, and miR-181a) were evaluated as prognostic and predictive factors of response to EGFR-TKI treatment in 39 advanced EGFR-mutated NSCLC patients who underwent EGFR-TKIs.

Firstly, we evaluated the correlation of basal miRNA expression with clinical characteristics of patients, thus documenting an inverse association between values of miR-21 and age, as previously reported [21].

Next, we explored the correlation of basal miRNA expression, defined as the level of circulating miRNA prior to EGFR-TKI, with survival outcomes and treatment response. Lacking a control group of matched healthy volunteers, high/low basal miRNA expression was established based on the median value for each miRNA. We did not find any statistically significant impact of basal miRNA expression on PFS and OS. However, higher basal values of miR-21 were documented in patients who achieved $P R / C R$ as best response compared to those with SD/PD $(p=0.011)$. Along the same line, patients who experienced a clinical benefit lasting at least six months displayed higher levels of circulating miR-21 $(p=0.039)$.

Dynamic variations of miRNA values after two months from the start of EGFR-TKI treatment (t1) were assessed to explore an early change of circulating miRNA expression as a way to predict differential EGFR-TKI response. Of note, at this time point, patients who experienced SD had an increase in miR-21 levels $(\mathrm{FC}=2.6)$ compared to patients achieving a response $(\mathrm{PR} / \mathrm{CR})(p=0.029)$. The same tendency was observed for miR-27a $(\mathrm{FC}=3.1)$ and miR-181a $(F C=2.0)$, although without reaching statistical significance.

However, when focusing on circulating levels of the candidate miRNAs at the time of the first radiological evaluation, we did not find any relevant difference in FC from basal. This evidence suggests that an early assessment of circulating miR-21 (t1) could be more informative for predicting the response to EGFR-TKIs than that performed at the time of the first radiological examination.

Similarly, no differences were found for miR-21, miR-27a, and miR-181a at the time of progression to EGFR-TKIs. In contrast, miR-21 was upregulated in gefitinib- and afatinibresistant NSCLC cells. In this regard, we could not exclude that the type of resistance mechanism to EGFR-TKI could have an impact on the levels of the candidate miRNAs, as most of our patients developed T790M resistance mutation at the time of PD, whereas cell lines harbored an EGFR-independent mechanism of resistance. Our preclinical findings are in line with the results of a recent study, showing that miR-21 was overexpressed in PC9 gefitinib-resistant (PC9R) cells that still had the EGFR mutation, but no T790M mutation [22]. Moreover, T790M EGFR-mutated NSCLC showed a different miRNA profile than tumors harboring L858R-activating mutations [23], suggesting a different role of miRNA expression in the resistance mechanisms in NSCLC cells harboring different EGFR mutations.

To date, this is the first study aimed at investigating the dynamic modification of circulating plasma miRNA levels as a predictor of response to different EGFR-TKIs (gefi- 
tinib, erlotinib, and afatinib) in advanced EGFR-mutated NSCLC. Previous research suggested that miR-21, miR-27a, and miR-181a may act as tumor-promoting miRNAs in NSCLC, and an increase in their circulating values could be associated with EGFR-TKI resistance $[10,11,22,24]$. The oncogenic properties of miR-21 in NSCLC can be explained by miR-21 target genes, which are involved in multiple pathways such as cell growth and proliferation, angiogenesis, invasion and metastasis, as also reported in recent extensive reviews and meta-analyses [24-26]. In particular, we demonstrated that the increase in miR-21 levels in our resistant cell lines was associated with increased phosphorylation of Akt. Conversely, downregulation of miR-21 resulted in less active signaling through the PI3-kinase-Akt pathway, rendering the cancer cells more susceptible to drug activity. Previous studies showed that miR-21 does indeed regulate the expression of PTEN and phosphorylation of its downstream kinase PI3-kinase-Akt pathway [27-31]. Moreover, the increase of phospho-Akt correlated with the reduction of drug-induced apoptosis and antitumor activity, suggesting that the Akt pathway plays a significant role in mediating drug resistance in different cancer cell models [30,32].

Our results are in line with these pieces of evidence, as an increase in miR-21 at t1 was associated with a lack of response to EGFR-TKIs, here intended as stability of disease. Accordingly, a trend of increased miR-27a and miR-181a in SD patients at $\mathrm{t} 1$ was apparent in our cohort, thus underlying their oncogenic properties. Of note, miR-21 is a well-known oncomiR in different tumors, and we demonstrated that miR-21 and miR-27 cooperatively inhibit a network of tumor suppressor genes involved with pancreatic tumor growth and progression [19]. Furthermore, previous studies suggested that plasma levels of miR-21 and miR-181 reflected tissue expression [33-35], and PCR-based analysis of pre- and postchemotherapy plasma samples showed that the downregulation of plasma miR-181a-5p predicted longer survival in pancreatic cancer patients [34].

Our results that high basal miR-21 levels were directly correlated with disease response and clinical benefit seem contradictory, but might be explained by the fact that miR-21 is a downstream effector of the activated EGFR signaling pathway. A statistically significant positive correlation was indeed observed between miR-21 expression levels and phosphoEGFR levels in NSCLC cell lines. These results are in agreement with previous findings in both NSCLC tissues and cell lines [36], and with the hypothesis that activator protein-1, which is activated by EGFR signaling [37], can in turn activate the miR-21 transcription through the binding to the promoter [38]. However, our study shows that miR-21 levels were modulated after treatment, and we hypothesize that a single-time assessment of circulating miRNA could be less adequate to predict treatment outcome compared to dynamic monitoring.

Our study has some limitations that could have influenced our results. First, we lacked a control group for assessing the relative expression of our candidate miRNAs in NSCLC patients compared to healthy individuals. Second, candidate miRNAs were arbitrarily selected based on available literature; miRNA PCR profiling in an initial discovery phase could have more accurately detected deregulated miRNAs. Moreover, our sample size was relatively small and heterogeneous, and this could have limited the detection of statistically significant differences, even considering the exploratory purpose of our research. However, our positive results surely deserve further investigation in a larger prospective study. Furthermore, a spike-in control was used as a reference for the relative miRNA quantification instead of internal controls. Although cel-miR-39 has been extensively used as a normalizer for circulating miRNA quantification, spike-in controls cannot normalize variations caused by factors prior to RNA isolation [39]. In addition, modulation of miR-21 might not only characterize cancer cells, but also be a common feature of pathological cell growth, as observed in mouse models with hypertrophic heart and other non-neoplastic diseases [40,41]. In this sense, we excluded a putative influence of comorbidities on miRNA circulating levels in our cohort of patients. Even though we did not perform concordance analysis between plasma and tissue levels, due to the lack of available material, we could assume that circulating levels reflected those detected in the primary tumor, as already 
reported [42]. Finally, since the third-generation EGFR-TKI osimertinib replaced earlygeneration EGFR-TKIs for the frontline treatment of metastatic EGFR-mutated NSCLC, our results need to be validated in this clinical setting as well. Nevertheless, the study setup and results can serve as a framework for further studies.

\section{Conclusions}

This study provides interesting hints about the role of dynamic changes of circulating miRNAs in predicting EGFR-TKI response in advanced EGFR-mutated NSCLC. Considering the minimal invasiveness of blood sampling, the evaluation of early circulating miRNA modification, especially miR-21, could indeed represent a useful tool for monitoring treatment outcome. Further extensive and prospective studies are warranted to confirm the predictive role of circulating miRNAs. These predictive biomarkers will then hopefully be applied in the clinical setting to select the best therapeutic approaches, thus circumventing unnecessary treatments and preventing collateral side effects.

Supplementary Materials: The following are available online at https:/ /www.mdpi.com/article/ 10.3390/cells10061520/s1, Figure S1: Kaplan Meier curves for central nervous system (CNS) metastases (A) and liver metastasis (B) and correlation with OS; Figure S2: Basal miRNA expression and correlation with gender (A), smoking status (B), age (C) and type of EGFR mutation (D); Figure S3. Basal miRNA expression and correlation with bone metastasis (A), central nervous system (CNS) metastasis (B), liver metastasis (C) and number of metastatic sites (D); Figure S4: Correlation between basal miRNA values; Figure S5: Modulation of miRNA expression at $\mathrm{t} 2$ and correlation with EGFRTKI response (A) and duration of clinical benefit (B); Table S1: Anti-proliferative effect of gefitinib, afatinib or osimertinib in NSCLC cells characterized for their EGFR and K-RAS status; Table S2: List of patients' comorbidities and correlation with miRNA levels.

Author Contributions: Conceptualization, A.L., E.G. and M.T.; methodology, E.G., M.C., R.M. and B.E.H.; formal analysis, A.L. and G.M.; investigation, A.A., A.G., A.L. and M.C.; resources, E.G., R.M., P.B., M.V. and M.T.; data curation, A.L., A.A., A.G., B.E.H. and P.B.; writing-original draft preparation, A.L.; writing—review and editing, A.L., M.C., G.M., R.M., A.G., P.B., B.E.H., F.P., M.V., M.T. and E.G.; supervision, M.T. and E.G.; project administration, A.L., R.M., M.T. and E.G.; funding acquisition, M.T. and E.G. All authors have read and agreed to the published version of the manuscript.

Funding: This work was supported by Associazione Italiana per la Ricerca sul Cancro (AIRC), Milan Grant IG2017-20074 (P.I. Marcello Tiseo), IG2020-24444 (P.I. Elisa Giovannetti), Dutch Cancer Society KWF grant\#11957 (P.I. Elisa Giovannetti).

Institutional Review Board Statement: The study was conducted according to the guidelines of the Declaration of Helsinki, and approved by the Institutional Review Board (or Ethics Committee) of University Hospital of Parma (protocol code 15/2019/TESS/AOUPR and date of approval 5 June 2019).

Informed Consent Statement: Informed consent was obtained from all subjects involved in the study.

Data Availability Statement: The data presented in this study are available on request from the corresponding author. The data of the patients are not publicly available due to privacy rules.

Acknowledgments: We thank Paraskevi Danae Veltsista, Bart Kok and Laura Meijer for direct technical help and fruitful discussion.

Conflicts of Interest: M.T. has been on advisory boards and received speakers' fees for Astra-Zeneca, Pfizer, Eli-Lilly, BMS, Novartis, Roche, MSD, Boehringer Ingelheim, Otsuka, Takeda, and Pierre Fabre. M.T. has received research grants from Astra-Zeneca and Boehringer Ingelheim. A.L. received speakers' fees for Astra-Zeneca. The funders had no role in the design of the study; in the collection, analyses, or interpretation of data; in the writing of the manuscript, or in the decision to publish the results.

\section{Appendix A. HGVS. Nomenclature of EGFR Mutations}

Exon 18

G719X: c.2155G > A p.(G719S); c.2155G > T p.(G719C); c.2156G > C p.(G719A). 
Ex19del: c.2235_2249del15; c.2235_2252 > AAT (complex); c.2236_2253del18; c.2237 _2251del15; c.2237_2254del18; c.2237_2255 > T (complex); c.2236_2250del15; c.2238_2255del18; c.2238_2248 > GC (complex); c.2238_2252 > GCA (complex); c.2239_2247del9; c.2239_2253del15; c.2239_2256del18; c.2239_2248TTAAGAGAAG > C(complex); c.2239_2258 > CA (complex); c.2240_2251del12; c.2240_2257del18; c.2240_2254del15; c.2239_2251 > C (complex).

Exon 20

Ins20: c.2307_2308ins9; c.2319_2320insCAC; c.2310_2311insGGT;

S768I: c.2303G > T p.(S768I);

T790M: c.2369C > T p.(T790M).

Exon 21

L858R: c.2573T > G p.(L858R).

\section{References}

1. $\quad$ Siegel, R.L.; Miller, K.D.; Fuchs, H.E.; Jemal, A. Cancer Statistics, 2021. CA Cancer J. Clin. 2021, 71, 7-33. [CrossRef]

2. Rosell, R.; Moran, T.; Queralt, C.; Porta, R.; Cardenal, F.; Camps, C.; Majem, M.; Lopez-Vivanco, G.; Isla, D.; Provencio, M.; et al. Screening for Epidermal Growth Factor Receptor Mutations in Lung Cancer. N. Engl. J. Med. 2009, 361, 958-967. [CrossRef]

3. Van Der Steen, N.; Giovannetti, E.; Carbone, D.; Leonetti, A.; Rolfo, C.D.; Peters, G.J. Resistance to epidermal growth factor receptor inhibition in non-small cell lung cancer. Cancer Drug Resist. 2018, 1, 230-249. [CrossRef]

4. Sequist, L.V.; Waltman, B.A.; Dias-Santagata, D.; Digumarthy, S.; Turke, A.B.; Fidias, P.; Bergethon, K.; Shaw, A.T.; Gettinger, S.; Cosper, A.K.; et al. Genotypic and Histological Evolution of Lung Cancers Acquiring Resistance to EGFR Inhibitors. Sci. Transl. Med. 2011, 3, 75ra26. [CrossRef]

5. Soria, J.-C.; Ohe, Y.; Vansteenkiste, J.; Reungwetwattana, T.; Chewaskulyong, B.; Lee, K.H.; Dechaphunkul, A.; Imamura, F.; Nogami, N.; Kurata, T.; et al. Osimertinib in Untreated EGFR-Mutated Advanced Non-Small-Cell Lung Cancer. N. Engl. J. Med. 2018, 378, 113-125. [CrossRef] [PubMed]

6. Leonetti, A.; Sharma, S.; Minari, R.; Perego, P.; Giovannetti, E.; Tiseo, M. Resistance mechanisms to osimertinib in EGFR-mutated non-small cell lung cancer. Br. J. Cancer 2019, 121, 725-737. [CrossRef]

7. Shenouda, S.K.; Alahari, S.K. MicroRNA function in cancer: Oncogene or a tumor suppressor? Cancer Metastasis Rev. 2009, 28, 369-378. [CrossRef]

8. Leonetti, A.; Assaraf, Y.G.; Veltsista, P.D.; El Hassouni, B.; Tiseo, M.; Giovannetti, E. MicroRNAs as a drug resistance mechanism to targeted therapies in EGFR-mutated NSCLC: Current implications and future directions. Drug Resist. Updates 2019, 42, 1-11. [CrossRef] [PubMed]

9. Han, F.; He, J.; Li, F.; Yang, J.; Wei, J.; Cho, W.C.; Liu, X. Emerging Roles of MicroRNAs in EGFR-Targeted Therapies for Lung Cancer. BioMed Res. Int. 2015, 2015, 672759. [CrossRef] [PubMed]

10. Wang, S.; Su, X.; Bai, H.; Zhao, J.; Duan, J.; An, T.; Zhuo, M.; Wang, Z.; Wu, M.; Li, Z.; et al. Identification of plasma microRNA profiles for primary resistance to EGFR-TKIs in advanced non-small cell lung cancer (NSCLC) patients with EGFR activating mutation. J. Hematol. Oncol. 2015, 8, 1-9. [CrossRef] [PubMed]

11. Ping, W.; Gao, Y.; Fan, X.; Li, W.; Deng, Y.; Fu, X. MiR-181a contributes gefitinib resistance in non-small cell lung cancer cells by targeting GAS7. Biochem. Biophys. Res. Commun. 2018, 495, 2482-2489. [CrossRef]

12. Livak, K.J.; Schmittgen, T.D. Analysis of Relative Gene Expression Data Using Real-Time Quantitative PCR and the $2-\Delta \Delta C T$ Method. Methods 2001, 25, 402-408. [CrossRef]

13. Asakage, M.; Usui, Y.; Nezu, N.; Shimizu, H.; Tsubota, K.; Yamakawa, N.; Takanashi, M.; Kuroda, M.; Goto, H. Comprehensive miRNA analysis using serum from patients with noninfectious uveitis. Investig. Ophthalmol. Vis. Sci. 2020, 61, 4. [CrossRef]

14. Ries, J.; Vairaktaris, E.; Kintopp, R.; Baran, C.; Neukam, F.W.; Nkenke, E. Alterations in miRNA expression patterns in whole blood of OSCC patients. Vivo 2014, 28, 851-862.

15. Ercan, D.; Zejnullahu, K.; Yonesaka, K.; Xiao, Y.; Capelletti, M.; Rogers, A.; Lifshits, E.; Brown, A.; Lee, C.; Christensen, J.G.; et al. Amplification of EGFR T790M causes resistance to an irreversible EGFR inhibitor. Oncogene 2010, 29, 2346-2356. [CrossRef] [PubMed]

16. Sciarrillo, R.; Wojtuszkiewicz, A.; Kooi, I.E.; Gómez, V.E.; Boggi, U.; Jansen, G.; Kaspers, G.-J.; Cloos, J.; Giovannetti, E. Using RNA-sequencing to Detect Novel Splice Variants Related to Drug Resistance in In Vitro Cancer Models. J. Vis. Exp. 2016, 2016, 54714. [CrossRef]

17. Heideman, D.A.M.; Thunnissen, F.B.; Doeleman, M.; Kramer, D.; Verheul, H.M.; Smit, E.F.; Postmus, P.E.; Meijer, C.J.L.M.; Meijer, G.A.; Snijders, P.J.F. A panel of high resolution melting (HRM) technology-based assays with direct sequencing possibility for effective mutation screening of EGFR and K-ras genes. Anal. Cell. Pathol. 2009, 31, 329-333. [CrossRef]

18. Giovannetti, E.; Funel, N.; Peters, G.J.; Del Chiaro, M.; Erozenci, L.A.; Vasile, E.; Leon, L.G.; Pollina, L.E.; Groen, A.; Falcone, A.; et al. MicroRNA-21 in Pancreatic Cancer: Correlation with Clinical Outcome and Pharmacologic Aspects Underlying Its Role in the Modulation of Gemcitabine Activity. Cancer Res. 2010, 70, 4528-4538. [CrossRef] 
19. Bianco, C.; Giovannetti, E.; Ciardiello, F.; Mey, V.; Nannizzi, S.; Tortora, G.; Troiani, T.; Pasqualetti, F.; Eckhardt, G.; De Liguoro, M.; et al. Synergistic antitumor activity of ZD6474, an inhibitor of vascular endothelial growth factor receptor and epidermal growth factor receptor signaling, with gemcitabine and ionizing radiation against pancreatic cancer. Clin. Cancer Res. 2006, 12, 7099-7107. [CrossRef] [PubMed]

20. Frampton, A.E.; Castellano, L.; Colombo, T.; Giovannetti, E.; Krell, J.; Jacob, J.; Pellegrino, L.; Roca-Alonso, L.; Funel, N.; Gall, T.M.H.; et al. MicroRNAs Cooperatively Inhibit a Network of Tumor Suppressor Genes to Promote Pancreatic Tumor Growth and Progression. Gastroenterology 2014, 146, 268-277.e18. [CrossRef] [PubMed]

21. Noren Hooten, N.; Fitzpatrick, M.; Wood, W.H.; De, S.; Ejiogu, N.; Zhang, Y.; Mattison, J.A.; Becker, K.G.; Zonderman, A.B.; Evans, M.K. Age-related changes in microRNA levels in serum. Aging 2013, 5, 725-740. [CrossRef] [PubMed]

22. Li, B.; Ren, S.; Li, X.; Wang, Y.; Garfield, D.; Zhou, S.; Chen, X.; Su, C.; Chen, M.; Kuang, P.; et al. MiR-21 overexpression is associated with acquired resistance of EGFR-TKI in non-small cell lung cancer. Lung Cancer 2014, 83, 146-153. [CrossRef]

23. Kim, J.Y.; Lee, W.J.; Park, H.Y.; Kim, A.; Shin, D.H.; Lee, C.H. Differential MicroRNA expression between EGFR T790M and L858R mutated lung cancer. J. Pathol. Transl. Med. 2018, 52, 275-282. [CrossRef]

24. Yuan, Y.; Xu, X.Y.; Zheng, H.G.; Hua, B.J. Elevated miR-21 is associated with poor prognosis in non-small cell lung cancer: A systematic review and meta-analysis. Eur. Rev. Med. Pharmacol. Sci. 2018, 22, 4166-4180.

25. Rolfo, C.; Fanale, D.; Hong, D.; Tsimberidou, A.; Piha-Paul, S.; Pauwels, P.; Meerbeeck, J.; Caruso, S.; Bazan, V.; Cicero, G.; et al. Impact of microRNAs in Resistance to Chemotherapy and Novel Targeted Agents in Non-Small Cell Lung Cancer. Curr. Pharm. Biotechnol. 2014, 15, 475-485. [CrossRef]

26. Pop-Bica, C.; Pintea, S.; Magdo, L.; Cojocneanu, R.; Gulei, D.; Ferracin, M.; Berindan-Neagoe, I. The Clinical Utility of miR-21 and let-7 in Non-small Cell Lung Cancer (NSCLC). A Systematic Review and Meta-Analysis. Front. Oncol. 2020, 10, 516850. [CrossRef] [PubMed]

27. Meng, F.; Henson, R.; Wehbe-Janek, H.; Ghoshal, K.; Jacob, S.T.; Patel, T. MicroRNA-21 Regulates Expression of the PTEN Tumor Suppressor Gene in Human Hepatocellular Cancer. Gastroenterology 2007, 133, 647-658. [CrossRef] [PubMed]

28. Meng, F.; Henson, R.; Lang, M.; Wehbe, H.; Maheshwari, S.; Mendell, J.T.; Jiang, J.; Schmittgen, T.D.; Patel, T. Involvement of Human Micro-RNA in Growth and Response to Chemotherapy in Human Cholangiocarcinoma Cell Lines. Gastroenterology 2006, 130, 2113-2129. [CrossRef] [PubMed]

29. Li, J.; Huang, H.; Sun, L.; Yang, M.; Pan, C.; Chen, W.; Wu, D.; Lin, Z.; Zeng, C.; Yao, Y.; et al. MiR-21 indicates poor prognosis in tongue squamous cell carcinomas as an apoptosis inhibitor. Clin. Cancer Res. 2009, 15, 3998-4008. [CrossRef]

30. Ding, S.; Zheng, Y.; Xu, Y.; Zhao, X.; Zhong, C. MiR-21/PTEN signaling modulates the chemo-sensitivity to 5-fluorouracil in human lung adenocarcinoma A549 cells. Int. J. Clin. Exp. Pathol. 2019, 12, 2339-2352. [PubMed]

31. Cavazzoni, A.; La Monica, S.; Alfieri, R.; Ravelli, A.; Van Der Steen, N.; Sciarrillo, R.; Madeddu, D.; Lagrasta, C.A.M.; Quaini, F.; Bonelli, M.; et al. Enhanced efficacy of AKT and FAK kinase combined inhibition in squamous cell lung carcinomas with stable reduction in PTEN. Oncotarget 2017, 8, 53068-53083. [CrossRef] [PubMed]

32. Lemke, L.E.; Paine-Murrieta, G.D.; Taylor, C.W.; Powis, G. Wortmannin inhibits the growth of mammary tumors despite the existence of a novel wortmannin-insensitive phosphatidylinositol-3-kinase. Cancer Chemother. Pharmacol. 1999, 44, 491-497. [CrossRef]

33. Khan, K.; Cunningham, D.; Peckitt, C.; Barton, S.; Tait, D.; Hawkins, M.; Watkins, D.; Starling, N.; Rao, S.; Begum, R.; et al. miR-21 expression and clinical outcome in locally advanced pancreatic cancer: Exploratory analysis of the pancreatic cancer Erbitux, radiotherapy and UFT (PERU) trial. Oncotarget 2016, 7, 12672-12681. [CrossRef] [PubMed]

34. Meijer, L.L.; Garajová, I.; Caparello, C.; Le Large, T.Y.S.; Frampton, A.E.; Vasile, E.; Funel, N.; Kazemier, G.; Giovannetti, E. Plasma miR-181a-5p downregulation predicts response and improved survival after FOLFIRINOX in pancreatic ductal adenocarcinoma. Ann. Surg. 2020, 271, 1137-1147. [CrossRef]

35. Arab, A.; Karimipoor, M.; Irani, S.; Kiani, A.; Zeinali, S.; Tafsiri, E.; Sheikhy, K. The Evaluation of miR-21 Level in Lung Tissue and Plasma of Nsclc Patients. Basic Clin. Cancer Res. 2017, 9, 13-21.

36. Seike, M.; Goto, A.; Okano, T.; Bowman, E.D.; Schetter, A.J.; Horikawa, I.; Mathe, E.A.; Jen, J.; Yang, P.; Sugimura, H.; et al. MiR-21 is an EGFR-regulated anti-apoptotic factor in lung cancer in never-smokers. Proc. Natl. Acad. Sci. USA 2009, 106, 12085-12090. [CrossRef]

37. Li, J.; Ma, C.; Huang, Y.; Luo, J.; Huang, C. Differential requirement of EGF receptor and its tyrosine kinase for AP-1 transactivation induced by EGF and TPA. Oncogene 2003, 22, 211-219. [CrossRef]

38. Fujita, S.; Ito, T.; Mizutani, T.; Minoguchi, S.; Yamamichi, N.; Sakurai, K.; Iba, H. miR-21 Gene Expression Triggered by AP-1 Is Sustained through a Double-Negative Feedback Mechanism. J. Mol. Biol. 2008, 378, 492-504. [CrossRef]

39. Schwarzenbach, H.; da Silva, A.M.; Calin, G.; Pantel, K. Data Normalization Strategies for MicroRNA Quantification. Clin. Chem. 2015, 61, 1333-1342. [CrossRef]

40. Thum, T.; Gross, C.; Fiedler, J.; Fischer, T.; Kissler, S.; Bussen, M.; Galuppo, P.; Just, S.; Rottbauer, W.; Frantz, S.; et al. MicroRNA-21 contributes to myocardial disease by stimulating MAP kinase signalling in fibroblasts. Nature 2008, 456, 980-984. [CrossRef]

41. Jenike, A.E.; Halushka, M.K. miR-21: A non-specific biomarker of all maladies. Biomark. Res. 2021, 9, 1-7. [CrossRef] [PubMed]

42. Zhao, Q.; Cao, J.; Wu, Y.C.; Liu, X.; Han, J.; Huang, X.C.; Jiang, L.H.; Hou, X.X.; Mao, W.M.; Ling, Z.Q. Circulating miRNAs is a potential marker for gefitinib sensitivity and correlation with EGFR mutational status in human lung cancers. Am. J. Cancer Res. 2015, 5, 1692-1705. [PubMed] 